\title{
A Robust Unknown Input Observer Design applied to an Industrial Pilot Plant ${ }^{\star}$
}

\author{
Matheus Senna de Oliveira* Renan Lima Pereira* \\ Anderson Tiago de Moraes* \\ * Instituto Tecnológico de Aeronáutica, Praça Marechal Eduardo \\ Gomes, Vila das Acácias, 12228-900 São José dos Campos, SP, Brazil, \\ (e-mails: \{matheus,renanrlp,andtiago\}@ita.br)
}

\begin{abstract}
This paper presents a robust unknown input observer (UIO) design procedure applied to an industrial pilot plant. The proposed design investigates the possible advantages of a proportional-integral UIO (PI-UIO) to solve the problem of states and disturbances estimation when the industrial plant is subject to parametric uncertainties. Existence conditions to obtain the PI-UIO structure are provided, as well as Linear Matrix Inequalities (LMIs) conditions to ensure the robust stability for the closed-loop system. A practical comparison using a proportional UIO widely used in the literature and the proposed approach is made. Finally, experimental results obtained with the application of the two UIOs strategies are presented.
\end{abstract}

Keywords: Discrete-time Systems; Unknown Input Observer; Industrial Pilot Plant; LMIs.

\section{INTRODUCTION}

In recent years, observer designs have been extensively studied in the control literature (Luenberger, 1971; Darouach et al., 1994; Ichalal and Mammar, 2015), where much attention has been focused on the problems of estimation of the states and unknown inputs (UIs). In particular, the problem of estimating UIs has been of great importance in practice since there are many situations where the disturbances or partial inputs are inaccessible. It stands out here the application in isolation and fault detection problems (Chang et al., 1997). Many different works regarding observer designs and existence conditions in systems with UIs were developed along the years (Darouach et al., 1994; Chang et al., 1997; Hassan et al., 2011). Hence, several strategies have been proposed concerning to UIs estimation using observers designs. In Tan and Edwards (2003), a sliding mode observer is used to handle a fault reconstruction problem. Rath et al. (2014) presented a performance analysis of vehicles suspensions using road profiles as unknown inputs. In Martinez et al. (2018), an observer design is presented for a wind turbine, subject to both actuator fault and disturbance.

Undoubtedly, an adequate approach to correctly estimate both states and unknown inputs is the so-called Unknown Input Observer (UIO). Although the origins of the UIOs can be found in early 80s (Watanabe et al., 1982), thereafter many papers have been proposed in order to improve estimation performance and robustness conditions (Chang et al., 1997; Hassan et al., 2011; Martinez et al., 2018). Due to its flexibility, this strategy can be applied to different classes of systems, such as descriptor systems, LPV systems, Takagi-Sugeno systems and uncertain systems (Bez-

\footnotetext{
* This study was financed in part by the Coordenação de Aperfeiçoamento de Pessoal de Nível Superior - Brasil (CAPES) - Finance Code 001 .
}

zaoucha et al., 2017; Ichalal and Mammar, 2015; Hassan et al., 2011; Takahashi and Peres, 1999).

In this sense, the main contribution of this paper consists in investigating a robust proportional-integral UIO (PIUIO) design procedure to estimate the states and the unknown inputs in a industrial plant when such system is subject to uncertainties caused by the encrusting of pipes. This type of plant is particularly interesting to allow students and researchers to do small scale experiments without the need of real and expansive industrial equipment (de Moraes and Kienitz, 2017). The proposed strategy is based on the observer presented in Shafai et al. (1996), which makes use of an explicit discrete-time integrator augmented structure, and in Chang et al. (1997), where the design of UIOs for linear systems with a proportional gain observer structure is addressed. The paper provides existence conditions for the presented observers, as well as a robust gain synthesis using Linear Matrix Inequalities (LMIs). In order to evaluate the PI-UIO performance, a practical comparison with a proportional UIO (P-UIO) widely used in the literature (Chang et al., 1997; Ichalal and Mammar, 2015) is performed in the experiment.

The remaining of this paper is organized as follows. Section 2 details the industrial pilot plant system and its uncertain linear model adopted. Sections 3 and 4 present, respectively, the proportional and proportional-integral UIO design procedures, detailing its synthesis conditions. Section 5 illustrates the effectiveness of the proposed approach for the case study. Section 6 concludes the paper.

Throughout the paper, the following notation is used: $\mathbb{R}^{n}$ is the $n$ dimensional Euclidean space; $A^{\top}$ denotes the transpose of matrix $A ; I_{n \times n}$ represents the identity of dimensions $n \times n$; the symbol $\star$ represents the transpose elements in the respective symmetric positions; $A^{\dagger}=$ $\left(A^{\top} A\right)^{-1} A^{\top}$ is the Moore-Penrose generalized inverse of $A$. 


\section{INDUSTRIAL PILOT PLANT}

Herein, a brief description of the industrial pilot-plant used is presented. Manufactured by Yokogawa for testing small scale industrial monitoring and control algorithms, the plant has 3 tanks: reserve tank (TQR), tank 1 (TQ1) and tank 2 (TQ2). Two pumps (B1 and B2) are responsible to transfer the fluid from TQR to TQ1 and TQ2. The outputs are the TQ1 and TQ2 levels (H1 and H2) and the inputs are the pumps flows (Q1 and Q2).

Figure 1 and 2 show, respectively, the industrial pilot plant and its schematic diagram. This paper addresses the tank level observation problem, therefore the controller used to operate the plant is not presented here. More details regarding this topic can be found in de Moraes and Kienitz (2017).

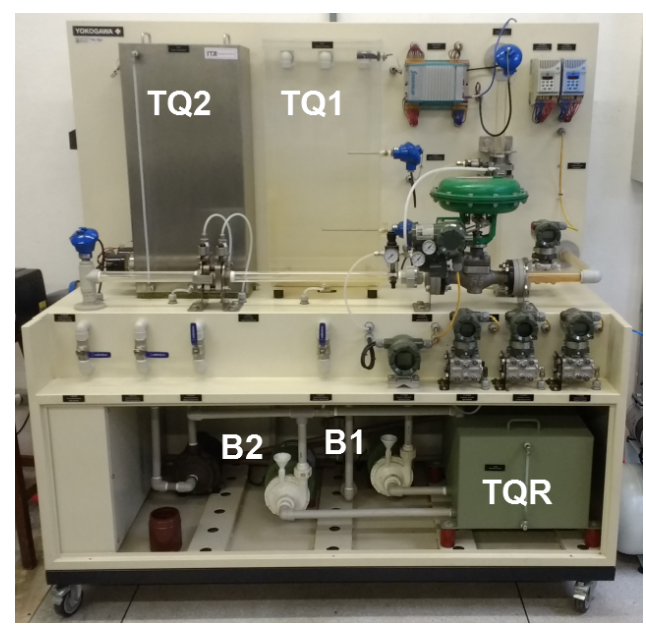

Figure 1. Industrial pilot plant.

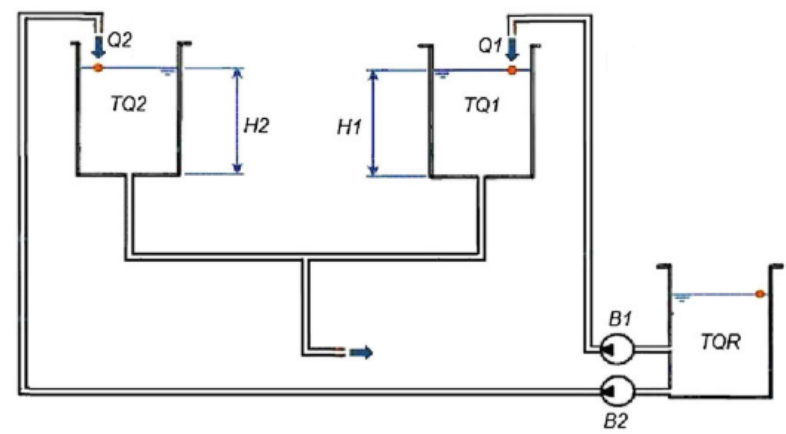

Figure 2. Schematic diagram of the industrial pilot plant.

The plant linear model holds around relevant operation points, obtained through the system identification. For the inputs, the flows are $50 \%$ and $48 \%$ for, respectively, pumps 1 and 2 . The outputs are defined as $13 \%$ for TQ1 and $21 \%$ for TQ2 levels. The model state vector is composed by the fluid loads and flows in TQ1 e TQ2. The uncertain model used in this paper is given by

$$
\begin{aligned}
\dot{x}(t) & =A_{c}(\rho) x(t)+B_{c} u(t)+E_{c} d(t), \\
y(t) & =C_{c}(\rho) x(t),
\end{aligned}
$$

where the state-space matrices are

$$
\begin{aligned}
A_{c}(\rho)= & {\left[\begin{array}{cccc}
0 & -8.78 \alpha_{1} & 0 & 0 \\
1.32 \alpha_{2} & -5.80 \rho_{1} & 0 & -8.03 \\
0 & 0 & 0 & -10.43 \alpha_{1} \\
0 & -5.14 & 1.32 \alpha_{2} & -11.18 \rho_{1}
\end{array}\right], } \\
B_{c} & =\left[\begin{array}{cccc}
1.58 \times 10^{-7} \times 0 & 0 & 0 \\
0 & 0 & 2.452 \times 10^{-7} & 0
\end{array}\right]^{\top}, \\
E_{c} & =\left[\begin{array}{cccc}
1.58 \times 10^{-7} & 0 & 0 & 0
\end{array}\right]^{\top}, \\
C_{c}(\rho) & =\left[\begin{array}{cccc}
1.318 \times 10^{5} \rho_{2} & 0 & 0 & 0 \\
0 & 0 & 1.318 \times 10^{5} \rho_{2} & 0
\end{array}\right],
\end{aligned}
$$

with $\alpha_{1}=10^{-8}$ and $\alpha_{2}=10^{5}$.

It is assumed a $10 \%$ parametric uncertainty due to the resistances presented as $\rho_{1}$, which are quite common because of the crusting of pipes. Moreover, it is considered a possible variation $\rho_{2}$ of $20 \%$ in $C_{c}$ structure.

The uncertain parameter vector $\rho \in \Theta$, with $\Theta$ being a hyper-rectangle defined by

$$
\Theta=\left\{\rho \in \mathbb{R}^{n_{\rho}} \mid \rho_{i} \in\left[\rho_{i}^{\min } \rho_{i}^{\max }\right]\right\},
$$

where $\rho_{i}^{\min }$ and $\rho_{i}^{\max }$ are, respectively, the lower and upper bounds of $\rho_{i}$. The parametric uncertainties can be described using a polytopic structure $\Phi$ given by

$$
\begin{aligned}
& \Phi=\left\{A_{c}(\rho)=\sum_{i=1}^{N_{\rho}} \mu_{i} A_{i} ; C_{c}(\rho)=\sum_{i=1}^{N_{\rho}} \mu_{i} C_{i}, \mu \in \Omega\right\}, \\
& \Omega=\left\{\mu \in R^{N_{\rho}}, \sum_{i=1}^{N_{\rho}} \mu_{i}=1, \mu_{i} \geq 0\right\}
\end{aligned}
$$

where $N_{\rho}=2^{n_{\rho}}$ is number of polytope vertices.

\section{ROBUST PROPORTIONAL UIO}

In this section, existence conditions and the main steps to obtain the robust proportional UIO (P-UIO) are provided. In order to clarify the main contributions, consider the following uncertain discrete-time system:

$$
\begin{aligned}
x(k+1) & =A(\rho) x(k)+B u(k)+E d(k), \\
y(k) & =C(\rho) x(k),
\end{aligned}
$$

where $x(k) \in \mathbb{R}^{n}$ is the state vector, $u(k) \in \mathbb{R}^{m}$ is the control input vector, $d(k) \in \mathbb{R}^{p}$ is the unknown input and $y(k) \in \mathbb{R}^{q}$ is the output vector. Matrices $B, E$ and the uncertain matrices $A(\rho)$ and $C(\rho)$ have appropriate dimensions.

The main contributions of this work are presented in the following section.

\subsection{P-UIO convergence conditions}

The proportional unknown input observer structure, as presented in Chang et al. (1997), is defined as follows:

$$
G_{P O}:\left\{\begin{array}{l}
z(k+1)=M z(k)+L y(k)+H u(k), \\
\widehat{x}(k)=z(k)-Q y(k),
\end{array}\right.
$$

where $z(k) \in \mathbb{R}^{n}$ is an auxiliary variable, $\widehat{x}(k) \in \mathbb{R}^{n}$ is the estimated states vector, $M=P A-L C$, with $P \in \mathbb{R}^{n \times n}$, $H \in \mathbb{R}^{n \times m}, Q \in \mathbb{R}^{n \times q}$ and the observer gain $L \in \mathbb{R}^{n \times q}$ are matrices with appropriate dimensions which must be obtained such that the estimation error $e(k)=x(k)-\widehat{x}(k)$ converges asymptotically to zero. 
It is possible to determine the P-UIO matrices using Lemma 1, where the observer convergence conditions are defined.

Lemma 1. Consider the P-UIO defined in (6). The estimation errors of the system (5) states and UI converge to zero if and only if the following conditions are satisfied:

- The pair $(P(\rho) A(\rho), C(\rho))$ is detectable in a quadratic sense $\forall \rho \in \Theta$;

- The system (5) has relative degree one, i.e. the matrices $C$ and $E$ satisfy

$$
\operatorname{rank}(C(\rho) E)=\operatorname{rank}(E)
$$

- The matrices $P, H$ and $Q$ are

$$
\left\{\begin{array}{l}
Q(\rho)=E(C(\rho) E)^{\dagger} \\
P(\rho)=I_{n \times n}-Q C(\rho) \\
H(\rho)=B-Q(\rho) C(\rho) B ;
\end{array}\right.
$$

- The matrix $(P(\rho) A(\rho)-L C(\rho))$ is quadratically stable $\forall \rho \in \Theta$.

Proof. The estimation error $e(k+1)$ dynamics, using (6), is given by

$$
\begin{aligned}
e(k+1)= & A(\rho) x(k)+E d(k)+B u(k)-L y(k) \\
& -(P(\rho) A(\rho)-L C(\rho)) \widehat{x}(k) \\
& -Q(\rho) C(\rho) A(\rho) x(k)-H(\rho) u(k) \\
& -Q(\rho) C(\rho) E d(k)-Q(\rho) C(\rho) B u(k) .
\end{aligned}
$$

After some manipulations, one obtains

$$
\begin{aligned}
e(k+1)= & (A(\rho)-Q(\rho) C(\rho) A(\rho)-L C(\rho)) x(k) \\
& -(P(\rho) A(\rho)-L C(\rho)) \widehat{x}(k) \\
& +(E-Q(\rho) C(\rho) E) d(k) \\
& +(B-Q(\rho) C(\rho) B-H(\rho)) u(k) .
\end{aligned}
$$

Applying the conditions derived from (8):

$$
\begin{aligned}
A-Q C A-L C & =P A-L C, \\
E-Q C E & =0, \\
H-B+Q C B & =0,
\end{aligned}
$$

then the error dynamics (10) becomes

$$
\begin{aligned}
e(k+1) & =M(\rho) e(k), \\
\widetilde{y}(k) & =y(k)-\widehat{y}(k)=C(\rho) e(k) .
\end{aligned}
$$

From the detectability condition, the matrix $L$ can be determined to guarantee the asymptotic convergence of the P-UIO estimation error to zero if the closed-loop matrix $M(\rho)=(P A)(\rho)-L C(\rho)$ is stable. Moreover, define the UI error as $e_{d}(k)=d(k)-\widehat{d}(k)$, which the UI signal can be estimated using the following relation (Chang et al., 1997):

$$
\begin{gathered}
\widehat{d}(k)=(C(\rho) E)^{\dagger}[ \\
y(k+1)-C(\rho) A(\rho) \widehat{x}(k) \\
-C(\rho) B u(k)] .
\end{gathered}
$$

If there exists an observer gain $L$ such that the system (10) is stable, then the estimation error

$$
e_{d}(k)=d(k)-\widehat{d}(k)=(C(\rho) E)^{\dagger} e(k),
$$

converges asymptotically to zero when $e(k) \rightarrow 0$. Hence, the proof is complete.

\subsection{Robust P-UIO Design}

The P-UIO robust gain synthesis, which guarantees the observer convergence for the uncertain system, is given by Theorem 2.
Theorem 2. Consider the uncertain discrete-time system (5) and the unknown input observer given in (6). There exists a P-UIO if there exists a positive definite matrix $X=X^{\top} \succ 0 \in \mathbb{R}^{n \times n}$ and a matrix $W \in \mathbb{R}^{n \times q}$ such that the following inequality problem holds

$$
\left[\begin{array}{cc}
X & \left(P_{j} A_{i}\right)^{\top} X-C_{i}^{\top} W^{\top} \\
\star & X
\end{array}\right] \succ 0,
$$

where the P-UIO observer gain is given by $L=X^{-1} W$.

Proof. Choosing as candidate Lyapunov function a quadratic function

$$
V(x(k))=x(k)^{\top} X x(k) .
$$

By the Lyapunov stability theory, if there exists a matrix $X=X^{\top} \succ 0$ that satisfies

$$
M(\rho) X M(\rho)^{\top}-X \prec 0,
$$

then the closed-loop P-UIO in (12) is stable, where $M(\rho)=$ $P(\rho) A(\rho)-L C(\rho)$. Applying the Schur complement in (17) results in

$$
\left[\begin{array}{cc}
X & (P(\rho) A(\rho)-L C(\rho))^{\top} X \\
\star & X
\end{array}\right] \succ 0 .
$$

Using the polytopic structure properties, we may obtain

$$
\sum_{j=1}^{N_{\rho}} \mu_{j} \sum_{i=1}^{N_{\rho}} \mu_{i}\left[\begin{array}{cc}
X & \left(P_{j} A_{i}-L C_{i}\right)^{\top} X \\
\star & X
\end{array}\right] \succ 0 .
$$

As $\sum_{j=1}^{N_{\rho}} \mu_{j}=\sum_{i=1}^{N_{\rho}} \mu_{i}=1$ and $W=L^{\top} X$, then the last inequation becomes equal to (15). Hence, the proof is complete.

\section{ROBUST PROPORTIONAL-INTEGRAL UIO}

In this section, existence conditions and the main steps to obtain the robust proportional-integral UIO (PI-UIO) for the uncertain discrete-time LPV system (5) are provided.

\subsection{PI-UIO convergence conditions}

Here, the proportional-integral unknown input observer (PI-UIO) combines the explicit integrator variable of Shafai et al. (1996) and the structure of Chang et al. (1997). Thus, the structure is formulated as follows:

$$
G_{P I O}:\left\{\begin{aligned}
& z(k+1)=(P A-L C) z(k)+L_{P} y(k) \\
& \quad+H u(k)+E v(k), \\
& v(k+1)=L_{I}(y(k)-\widehat{y}(k))+v(k), \\
& \widehat{x}(k)=z(k)-Q y(k),
\end{aligned}\right.
$$

where $v(k) \in \mathbb{R}^{p}$ is the integrator variable, $P \in \mathbb{R}^{n \times n}$, $H \in \mathbb{R}^{n \times m}, Q \in \mathbb{R}^{n \times q}$, the proportional gain $L_{P} \in \mathbb{R}^{n \times q}$ and the integral gain $L_{I} \in \mathbb{R}^{p}$ are matrices with appropriate dimensions which must be obtained such that the estimation error $e(k)=x(k)-\widehat{x}(k)$ converges asymptotically to zero.

It is possible to determine the PI-UIO matrices using Lemma 3, where the convergence conditions of the observer are defined similarly to the P-UIO case.

Lemma 3. Consider the PI-UIO defined in (20). The estimation errors of the system (5) states and UI converge to zero if and only if the following conditions are satisfied:

- The pair $(\mathcal{A}(\rho), \mathcal{C}(\rho))$ is detectable in a quadratic sense $\forall \rho \in \Theta$; 
- The system (5) has relative degree one, i.e. the matrices $C$ and $E$ satisfy

$$
\operatorname{rank}(C(\rho) E)=\operatorname{rank}(E),
$$

- The matrices $P, H$ and $Q$ are

$$
\left\{\begin{array}{l}
Q(\rho)=E(C(\rho) E)^{\dagger} \\
P(\rho)=I_{n \times n}-Q(\rho) C(\rho) \\
H(\rho)=B-Q(\rho) C(\rho) B
\end{array}\right.
$$

- The matrix $\left(\mathcal{A}(\rho)-L_{a} \mathcal{C}(\rho)\right)$ is quadratically stable $\forall \rho \in \Theta$.

Proof. The estimation error $e(k+1)$ dynamics, using $(20)$, is given by

$$
\begin{aligned}
e(k+1)= & A(\rho) x(k)+E d(k)+B u(k)-L_{P} y(k) \\
& -\left(P(\rho) A(\rho)-L_{P} C(\rho)\right) \widehat{x}(k)-E v(k) \\
& -Q(\rho) C(\rho) A(\rho) x(k)-H(\rho) u(k) \\
& -Q(\rho) C(\rho) E d(k)-Q(\rho) C(\rho) B u(k) .
\end{aligned}
$$

After some manipulations, one obtains

$$
\begin{aligned}
e(k+1)= & \left(A(\rho)-Q(\rho) C(\rho) A(\rho)-L_{P} C(\rho)\right) x(k) \\
& -\left(P(\rho) A(\rho)-L_{P} C(\rho)\right) \widehat{x}(k) \\
& +(E-Q(\rho) C(\rho) E) d(k)-E v(k) \\
& +(B-Q(\rho) C(\rho) B-H(\rho)) u(k) .
\end{aligned}
$$

Applying the conditions derived from (22)

$$
\begin{aligned}
A-Q C A-L C & =P A-L C, \\
E-Q C E & =0, \\
H-B+Q C B & =0,
\end{aligned}
$$

then the error dynamics $(24)$ becomes

$$
\begin{aligned}
e(k+1) & =\mathcal{M}(\rho) e(k)-E v(k), \\
\widetilde{y}(k) & =y(k)-\widehat{y}(k)=C e(k),
\end{aligned}
$$

where $\mathcal{M}(\rho)=(P A)(\rho)-L_{P} C(\rho)$. Now, let us define an augmented error vector $e_{a}(k)=\left[e(k)^{\top} v(k)^{\top}\right]^{\top}$. Considering the system (20), the integrator term may be rewritten as

$$
\begin{aligned}
v(k+1) & =L_{I}(y-\widehat{y}(k))+v(k) \\
& =L_{I} C(\rho) \underbrace{(x(k)-\widehat{x}(k))}_{e(k)}+v(k) .
\end{aligned}
$$

Therefore, the PI-UIO augmented error dynamics, considering the polytopic representation, becomes

$$
e_{a}(k+1)=\sum_{j=1}^{N_{\rho}} \mu_{j} \sum_{i=1}^{N_{\rho}} \mu_{i}\left(\left[\begin{array}{ll}
\mathcal{M}_{i, j} & -E \\
L_{I} C_{i} & I_{p \times p}
\end{array}\right] e_{a}(k)\right) .
$$

Recalling that $\mathcal{M}(\rho)=(P A)(\rho)-L_{P} C(\rho)$ and after some mathematical manipulation, the previous equation may be rewritten as

$$
\begin{aligned}
e_{a}(k+1)=\sum_{j=1}^{N_{\rho}} \mu_{j} \sum_{i=1}^{N_{\rho}} \mu_{i} & (\underbrace{\left[\begin{array}{cc}
P_{j} A_{i} & -E \\
0 & I_{p \times p}
\end{array}\right]}_{\mathcal{A}_{i, j}} \\
& -\underbrace{\left[\begin{array}{c}
L_{P} \\
L_{I}
\end{array}\right]}_{L_{a}} \underbrace{\left[\begin{array}{ll}
C_{i} & 0
\end{array}\right]}_{\mathcal{C}_{i}}) e_{a}(k),
\end{aligned}
$$

which can be reorganized as

$$
e_{a}(k+1)=\sum_{j=1}^{N} \mu_{j} \sum_{i=1}^{N} \mu_{i}\left(\mathcal{A}_{i, j}-L_{a} \mathcal{C}_{i}\right) e_{a}(k) .
$$

From the detectability condition $(\mathcal{A}, \mathcal{C})$, the matrix $L_{a}$ can be determined to guarantee the asymptotic convergence of the PI-UIO estimation error if the closed-loop matrix $\left(\mathcal{A}_{i, j}-L_{a} \mathcal{C}_{i}\right)$ is stable. The disturbance estimation error convergence follows the same idea from the P-UIO. Thus, the proof is complete.

\subsection{Robust PI-UIO Design}

The PI-UIO robust gain synthesis, which guarantees the observer convergence for the uncertain system, is given by Theorem 4.

Theorem 4. Consider the uncertain discrete-time system (5) and the unknown input observer given in (20). There exists a PI-UIO if there exists a positive definite matrix $X=X^{\top} \succ 0 \in \mathbb{R}^{(n+p) \times(n+p)}$ and a matrix $W \in \mathbb{R}^{(n+p) \times q}$ such that the following inequality problem holds

$$
\left[\begin{array}{cc}
X & \mathcal{A}_{i, j}^{\top} X-\mathcal{C}_{i}^{\top} W^{\top} \\
\star & X
\end{array}\right] \succ 0
$$

where the PI-UIO observer gain is given by $L_{a}=X^{-1} W$.

Proof. The proof is straightforward using the same procedure presented in Theorem 2. The only difference comes from the existence conditions given in (30).

\section{EXPERIMENTAL RESULTS}

In order to evaluate the effectiveness of the proposed UIOs, an experiment was performed in the industrial pilot plant described in Section 2. Regarding the continuoustime model discrezation, some methods present technical difficulties, such as the exponential dependency on the uncertain parameter for the zero-order hold ( $\mathrm{ZOH}$ ) (Tóth et al., 2010). Therefore, the matrices of the uncertain continuous-time system (1) were discretized using the forward Euler method with a sample time of 0.01 [s].

The plant was first controlled to achieve the levels of $13 \%$ and $21 \%$ for, respectively, tanks 1 and 2 , which are the equilibrium points for the plant linear model. At 600 seconds, the references were increased in 5\% $(18 \%$ and $26 \%$ ) for both outputs. The unknown input was qualitatively set at approximately 1000 seconds in tank 1. It consists in a rectangular signal with duration of 400 seconds and magnitude of approximately 0.1 liters per second (or 10\%).

The robust UIOs gains were calculated using Theorems 2 and 4, whose LMIs were programmed using the Yalmip package (Lofberg, 2004) and solved using the toolbox SeDuMi (Sturm, 1999) in MATLAB 9.5 (2018b). The P$\mathrm{UIO}$ and PI-UIO gains obtained are, respectively:

$L=10^{-2}\left[\begin{array}{cc}-0.000 & -0.000 \\ 0.706 & -0.012 \\ 0.000 & 0.000 \\ 0.126 & 0.010\end{array}\right], L_{a}=10^{-2}\left[\begin{array}{cc}-0.000 & -0.000 \\ 0.715 & -0.03 \\ 0.000 & 0.000 \\ 0.119 & 0.010 \\ 0.000 & 0.000\end{array}\right]$

Figure 3 presents the estimation error signals for the output 1 . It is possible to notice the better performance of the PI-UIO than the P-UIO during all the experiment.

The error signals for the output 2 are depicted in Figure 4. One may note that the estimation results are similar for both UIOs. 


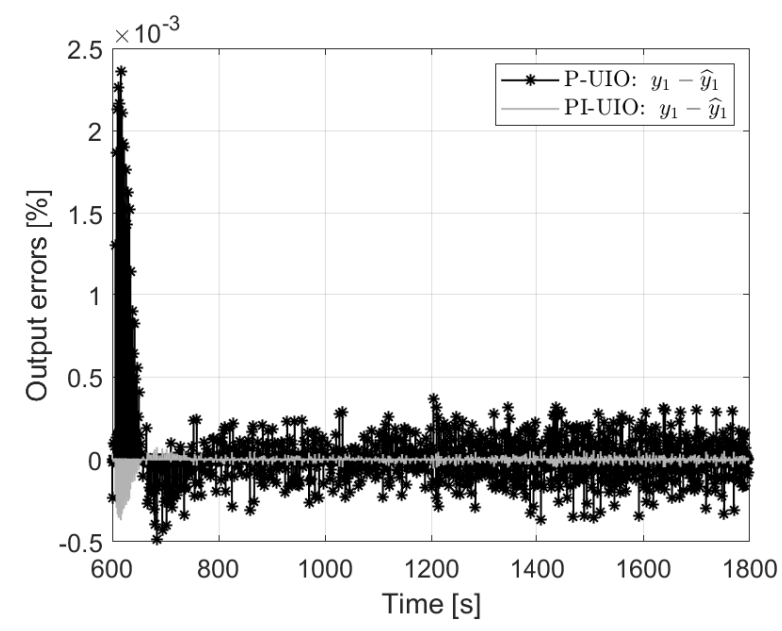

Figure 3. Error signals of the P-UIO and PI-UIO for output 1.

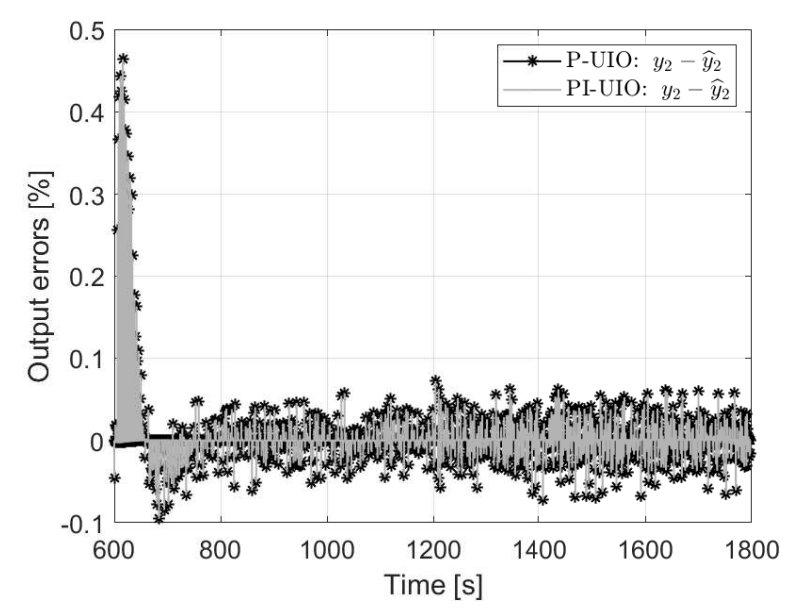

Figure 4. Error signals of the P-UIO and PI-UIO for output 2.

Figure 5 presents the unknown input estimation signal for both observers. In order to reduce the effect of noise in the estimations, a moving average filter with window size of 100 points was applied to the signals. As expected, the UIOs estimations depicts a positive step with magnitude of approximately $10 \%$ starting in 1000 seconds, which returns to its initial value when the unknown input is removed after 400 seconds. Moreover, it is possible to note a small DC error, which may be addressed to the non-modeled dynamics in the industrial pilot plant model. Without the use of the UIO, it would be difficult to detect such disturbance, which can done thanks to the decoupling capacity of the unknown input observers.

In order to evaluate the comparison, the integral absolute error (IAE) criteria was used. It may be obtained using the following relation:

$$
\mathrm{IAE}=\sum_{k=1}^{N}|y(k)-\widehat{y}(k)|,
$$

where $N$ is the size of the discrete-time vectors $y(k)$ and $\widehat{y}(k)$.

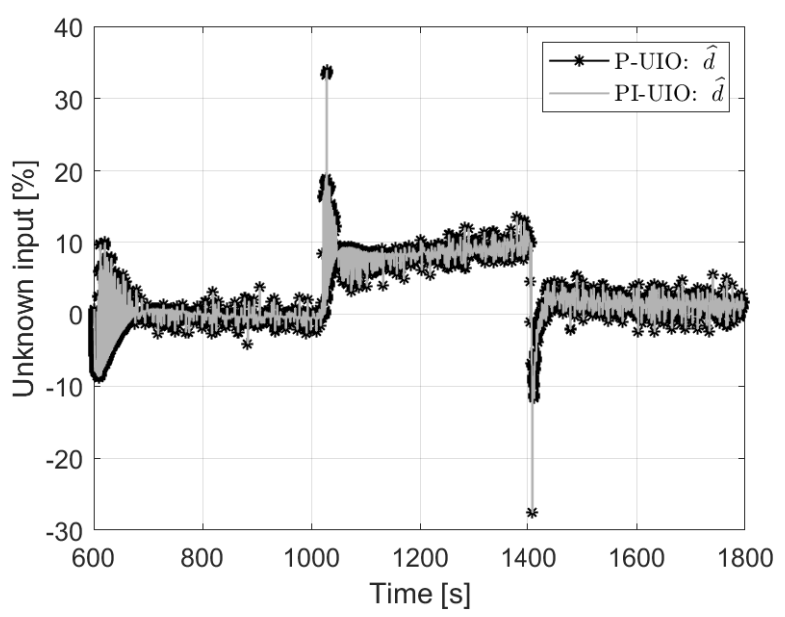

Figure 5. Estimated unknown input for the P-UIO and PI-UIO.

From Table 1, it is possible to note that the PI-UIO is able to achieve a better performance than the proportional case for both outputs. Moreover, one may note that the output 1 , which is directly affected by the unknown input, presented more significant results in favor to the proportional-integral observer.

Table 1. IAE results for both P-UIO and PIUIO structures.

\begin{tabular}{ccc}
\multirow{2}{*}{ Structure } & \multicolumn{2}{c}{ IAE values } \\
\cline { 2 - 3 } & $y_{1}-\widehat{y_{1}}$ & $y_{2}-\widehat{y_{2}}$ \\
\hline P-UIO & 0.3526 & 66.623 \\
PI-UIO & $\mathbf{0 . 0 9 7 4}$ & $\mathbf{6 6 . 4 5 9}$ \\
\hline
\end{tabular}

\section{CONCLUSION}

This paper investigated the possible advantages of a robust proportional-integral UIO (PI-UIO) applied to an industrial pilot plant subject to an imprecise model caused by the encrusting of pipes. The investigation aimed to solve the problem of estimating states and unknown input of the system. Existence conditions for the PI-UIO were presented, as well a set of Linear Matrix Inequalities used to obtain the observer gain that ensures the robust stability for the closed-loop system. In order to evaluate the effectiveness of the proposed approach, a comparison with the proportional case was performed. Experimental results highlighted a better performance of the proportionalintegral unknown input observer, which also was able to adequately estimate the unknown input. Future works may regard a different modeling of the system and the use of extra sensors to adequately analyze the observer performance for all system variables. Moreover, less conservative stability conditions may also be addressed.

\section{REFERENCES}

Bezzaoucha, S., Voos, H., and Darouach, M. (2017). A Polytopic Observer Design Approach for Landing Control of a Quadrotor UAV. IFAC-PapersOnLine, 50(1), 9753-9759. doi:10.1016/J.IFACOL.2017.08.2180.

Chang, S.K., You, W.T., and Hsu, P.L. (1997). Design of general structured observers for linear systems with 
unknown inputs. Journal of the Franklin Institute, 334(2), 213-232. doi:10.1016/S0016-0032(96)00077-4.

Darouach, M., Zasadzinski, M., and Xu, S. (1994). Fullorder observers for linear systems with unknown inputs. IEEE Transactions on Automatic Control, 39(3), 606609. doi:10.1109/9.280770.

de Moraes, A.T. and Kienitz, K.H. (2017). Robust control of an industrial pilot plant. IEEE Latin America Transactions, 15(9), 1613-1620. doi:10.1109/TLA.2017. 8015043.

Hassan, L., Zemouche, A., and Boutayeb, M. (2011). Hinfinity unknown input observers design for a class of nonlinear time-delay systems. CD-ROM.

Ichalal, D. and Mammar, S. (2015). On Unknown Input Observers for LPV Systems. IEEE Transactions on Industrial Electronics, 62(9), 5870-5880. doi:10.1109/ TIE.2015.2448055.

Lofberg, J. (2004). YALMIP : a toolbox for modeling and optimization in MATLAB. In 2004 IEEE International Conference on Robotics and Automation (IEEE Cat. No.04CH37508), 284-289. IEEE. doi:10.1109/CACSD. 2004.1393890.

Luenberger, D.G. (1971). An Introduction to Observers. IEEE Transactions on Automatic Control, 16(6), 596602. doi:10.1109/TAC.1971.1099826.

Martinez, J.J., Loukkas, N., and Meslem, N. (2018). Hinfinity set-membership observer design for discretetime LPV systems. International Journal of Control, 1-25. doi:10.1080/00207179.2018.1554910.

Rath, J.J., Veluvolu, K.C., and Defoort, M. (2014). Adaptive super-twisting observer for estimation of random road excitation profile in automotive suspension systems. TheScientific WorldJournal, 2014, 203416. doi: $10.1155 / 2014 / 203416$.

Shafai, B., Beale, S., Niemann, H., and Stoustrup, J. (1996). LTR design of discrete-time proportionalintegral observers. IEEE Transactions on Automatic Control, 41(7), 1056-1062. doi:10.1109/9.508916.

Sturm, J.F. (1999). Using SeDuMi 1.02, A Matlab toolbox for optimization over symmetric cones. Optimization Methods and Software, 11(1-4), 625-653. doi:10.1080/ 10556789908805766.

Takahashi, R. and Peres, P. (1999). Unknown input observers for uncertain systems: a unifying approach and enhancements. In Proceedings of 35th IEEE Conference on Decision and Control, volume 2, 1483-1488. IEEE. doi:10.1109/CDC.1996.572726.

Tan, C.P. and Edwards, C. (2003). Sliding mode observers for robust detection and reconstruction of actuator and sensor faults. International Journal of Robust and Nonlinear Control, 13(5), 443-463. doi:10.1002/rnc.723.

Tóth, R., Heuberger, P., and Van den Hof, P. (2010). Discretisation of linear parameter-varying state-space representations. IET Control Theory \& Applications, 4(10), 2082-2096. doi:10.1049/iet-cta.2009.0572.

Watanabe, K., Himmelblau, and D. M. (1982). Instrument fault detection in systems with uncertainties. International Journal of Systems Science, 13(2), 137-158. doi: 10.1080/00207728208926337. 